Check for updates

Cite this: Phys. Chem. Chem. Phys., 2021, 23, 12490

Received 12th March 2021,

Accepted 7th May 2021

DOI: $10.1039 / \mathrm{d} 1 \mathrm{cp} 01122 \mathrm{~g}$

rsc.li/pccp

\section{Comment on "On the thermodynamic stability of bubbles, immiscible droplets, and cavities" by G. S. Manning, Phys. Chem. Chem. Phys., 2020, 22, 17523}

\begin{abstract}
Lasse Makkonen and Tapio Vehmas (D) *
In this comment, the thermodynamic analysis of the stability of nanobubbles is discussed in reference to the recent paper by Manning (G. S. Manning, On the Thermodynamic Stability of Bubbles,Immiscible Droplets, and Cavities, Phys. Chem. Chem. Phys., 2020, 22, 17523-17531). It is argued that Manning's critcism on the classical Epstein-Plesset model of bubble stability is unwarranted, and that the Young-Laplace-equation must be understood as a fundamental law of the pressure difference across a curved interface regardless of the reaction of the gas in the bubble. Consequently, the internal pressure and the radius of a bubble are inherently linked, so that the net force considered in Manning's stability analysis does not exist.
\end{abstract}

Recently in this journal, Manning ${ }^{1}$ presented a paper "On the thermodynamic stability of bubbles, immiscible droplets, and cavities" where he concluded that the Epstein-Plesset model of bubble dissolution is inapplicable and claimed to give a direct proof that nanobubbles are unstable if the surface tension is assumed independent of sphere radius. These conclusions were described as representing "a sharper understanding of the Laplace equation and the physics underlying it". We find Manning's analysis and his interpretation of the Young-Laplace equation misleading and, therefore, wish to submit the following comments.

First, we would like to emphasize that Manning's stability analysis ${ }^{1}$ has very little to do with the Epstein-Plesset model, ${ }^{2}$ which deals with dissolution of gas from a bubble to the surrounding liquid. The Epstein-Plesset model is a diffusion calculation and does not take a stand on bubble stability criteria. Results similar to Epstein and Plesset were obtained by Lunggren and Eriksson, ${ }^{3}$ who made calculations related more specifically to nanosized bubbles.

Manning ${ }^{1}$ analyses the stability of a bubble from which no gas escapes. This, of course, rules out the applicability of the Epstein-Plesset diffusion model, which predicts stable bubbles when assuming zero gas solvability. Manning's ${ }^{1}$ argument is that, since the pressure gradient is discontinuous across the gas-liquid interface, the concentration gradient cannot be continuous, and, therefore, Henry's law does not relate the internal gas pressure of a bubble to the dissolved gas concentration just outside the bubble. However, such an assumption is not necessary for the calculations by Epstein and Plesset or

VTT Technical Research Centre of Finland, Box 1000, 02044 VTT, Espoo, Finland. E-mail: tapio.vehmas@vtt.fi; Tel: +358207224618
Ljunggren and Eriksson. Rather, the assumption is that the system is in chemical equilibrium, i.e. "Full contact equilibrium prevails and the chemical potentials are the same everywhere", as stated by Ljunggren and Eriksson. ${ }^{3}$

A theoretical framework of a bubble in chemical equilibrium was given by Ward et al. ${ }^{4}$ In order to derive equations for the bubble behaviour, four different chemical potentials must be considered. These are the chemical potential of water outside the bubble $\left(\mu_{1}{ }^{\prime}\right)$, vapour pressure inside the bubble $\left(\mu_{1}{ }^{\prime \prime}\right)$, dissolved gas concentration outside the bubble $\left(\mu_{2}{ }^{\prime}\right)$ and pure gas pressure inside the bubble $\left(\mu_{2}^{\prime \prime}\right)$. These four chemical potentials can be derived from the standard chemical potentials of the water $\left(\mu_{1}^{0}\left(P_{\infty}, T\right)\right)$ and gas $\left(\mu_{2}^{0}\left(P^{\prime}, T\right)\right)$ :

$$
\begin{gathered}
\mu_{1}{ }^{\prime}=\mu_{1}^{0}\left(P_{\infty}, T\right)+\nu_{1}{ }^{\prime}\left(p_{0}-p_{\infty}\right) \\
\mu_{2}{ }^{\prime}=\mu_{2}^{0}\left(P^{\prime}, T\right)+k T \ln \frac{c^{\prime}}{c_{0}} \\
\mu_{1}{ }^{\prime \prime}=\mu_{1}^{0}\left(P_{\infty}, T\right)+k T \ln \frac{p_{1}{ }^{\prime \prime}}{p_{\infty}} \\
\mu_{2}^{\prime \prime}=\mu_{2}^{0}\left(P^{\prime}, T\right)+k T \ln \frac{p_{2}{ }^{\prime \prime}}{p_{0}}
\end{gathered}
$$

With $\nu_{1}{ }^{\prime}=$ molar volume of the water, $p_{0}=$ pressure outside the bubble, $p_{\infty}=$ water vapour pressure in the bulk liquid defined at the planar interface at the pressure $p_{0}, c^{\prime}=$ dissolved gas concentration in the bulk solution, $c_{\mathrm{o}}=$ saturated gas dissolution concentration, $p_{1}{ }^{\prime \prime}=$ vapour pressure inside the bubble and $p_{2}{ }^{\prime \prime}=$ gas pressure inside the bubble. Note, that there are errors in the paper by Ward ${ }^{4}$ in these equations. 
In chemical equilibrium, chemical potential of the water and vapour must be equal. Chemical potential of the vapour inside the bubble reduces to the Kelvin factor $\eta$ (eqn (5)). Moreover, the chemical potential of the gas phase is the same inside and outside the bubble (eqn (6)).

$$
\begin{gathered}
\mu_{1}^{\prime}=\mu_{1}^{\prime \prime}(=) \frac{p_{1}^{\prime \prime}}{p_{\infty}}=\mathrm{e}^{\frac{v_{1}^{\prime}\left(p^{\prime}-p_{\infty}\right)}{k T}}=\eta \\
\mu_{2}^{\prime}=\mu_{2}^{\prime \prime}(=) \frac{c^{\prime}}{c_{0}}=\frac{p_{2}^{\prime \prime}}{p_{0}}
\end{gathered}
$$

The total pressure inside the bubble is the sum of vapour pressure and gas pressure:

$$
p^{\prime \prime}=p_{1}^{\prime \prime}+p_{2}^{\prime \prime}=p_{\infty} \eta+\frac{c^{\prime}}{c_{0}} p_{0}
$$

According to Laplace-Young equation, the pressure inside the bubble depends on the surface tension $\sigma$ and bubble radius $r$ :

$$
p^{\prime \prime}-p_{0}=\frac{2 \sigma}{r}
$$

By substituting the internal pressure by the individual terms of vapour pressure and gas pressure, given above, the equilibrium radius $\left(r_{\mathrm{e}}\right)$ for a bubble can be presented as

$$
\frac{2 \sigma}{r}+p_{0}=p_{\infty} \eta+\frac{c^{\prime}}{c_{0}} p_{0}(=) r_{\mathrm{e}}=\frac{2 \sigma}{p_{\infty} \eta+\frac{c^{\prime}}{c_{0}} p_{0}-p_{0}}
$$

Solving eqn (9) shows that the range of $r_{\mathrm{e}}$ is such that in water at atmospheric pressure, $r_{\mathrm{e}}=250 \mu \mathrm{m}$ at $c^{\prime} / c_{0}=1$. The equilibrium is unstable, so that bubbles larger than $r_{\mathrm{e}}$ grow and those smaller than $r_{\mathrm{e}}$ dissolve. No solution exists below $c^{\prime} / c_{0}=0.9939$. Accordingly, under normal temperature and pressure (NTP), nanobubbles cannot be in thermodynamic equilibrium with water. Only at a high supersaturation and a high pressure, can suspended nanobubbles prevail according to this theory. The limiting radius of eqn (9), without the vapour pressure term, can be deduced, also from the classical theory by EpsteinPlesset $^{2}$. Consequently, the present theoretical prediction is that nanobubbles in natural conditions are inherently unstable. In eqn (9), Henry's law is applied only to relate the saturated gas solution concentration $c_{\mathrm{o}}$ to the external pressure.

Our second comment concerns the fundamental understanding of the Young-Laplace equation. Manning ${ }^{1}$ refers to the basic thermodynamic derivation of the Young-Laplace equation. However, there exists also a mechanical derivation, ${ }^{5,6}$ the implications of which need to be understood. The normal stress balance for fluids meeting at an interface is

$$
\Delta P=\nabla \cdot \tilde{n}=\gamma \cdot H=\gamma\left(1 / R_{1}+1 / R_{2}\right)
$$

where $\tilde{n}$ is the unit normal pointing to the surface, $H$ is the mean curvature, $R_{1}$ and $R_{2}$ are the principal radii of curvature, and $\gamma$ is the surface tension. For a bubble, $\Delta P=P_{\mathrm{b}}-P_{\mathrm{w}}$, i.e., the difference between gas pressure in the bubble $P_{\mathrm{b}}$ and liquid pressure outside the bubble $P_{\mathrm{w}}$, and $R_{1}=R_{2}$ equals the bubble radius $R$. The Young-Laplace equation for a bubble then follows as

$$
\Delta P=2 \gamma / R
$$

It is noteworthy that this derivation does not involve any changes in volume. Thus, eqn (11) is widely applicable, for example, to a bubble containing incompressible gas. According to the mechanical derivation, the Young-Laplace equation simply represents a statement that the pressure $2 \gamma / R$ is exerted by the liquid to the bubble.

As pointed out above, the mechanical derivation of YoungLaplace equation has nothing to do with how the gas in a bubble behaves. Accordingly, the force exerted by the gas in the bubble to the liquid is not an active force that could affect the realization of eqn (11) in any way. It is a reaction force for the radial surface tensional force exerted on the bubble.

By the third law of Newton's classical mechanics, all forces occur in pairs such that, if one object exerts a force on another object, then the second object exerts an equal and opposite reaction force on the first. ${ }^{7}$ However, the terms 'action' and 'reaction' have the misleading suggestion of causality. It is, therefore, easy to think of the second force as being there because of the first, and even happening some time after the first. This is incorrect; the forces are simultaneous, and are there for the same reason. ${ }^{8}$

An essential ingredient of Manning's ${ }^{1}$ analysis is "the net force exerted by the surroundings of a bubble, including the surface, on the interior of the bubble". Of that concept, he writes: "If this net force is directed inwards, the bubble shrinks. If it is directed outwards, the bubble expands. If it vanishes, the bubble is in a state of mechanical equilibrium. The Laplace equation, eqn (6), is the condition for vanishing net force. From this condition, we recognize that the net zero force is the resultant of two competing tendencies." As pointed out above, this way of thinking is misleading. The action force and the reaction force are inherently linked and simultaneous, even in a dynamic situation. Therefore, no such net force, as postulated by Manning, ${ }^{1}$ can exist.

For the same reason, "an arbitrary constant pressure difference, independent of $R$ ", used by Manning ${ }^{1}$ in the derivation of the stability condition, is an inappropriate concept. The pressure difference cannot be arbitrary, because it is set by eqn (11) exactly at all times.

Manning ${ }^{1}$ presents his eqn (2) for the change in the Helmholtz free energy $F$ as

$$
\mathrm{d} F=-P_{\mathrm{w}} \mathrm{d} V_{\mathrm{w}}-P_{\mathrm{b}} \mathrm{d} V_{\mathrm{b}}+\gamma(R) \mathrm{d} A
$$

Since $^{1} \mathrm{~d} V_{\mathrm{w}}=-\mathrm{d} V_{\mathrm{b}}$, eqn (3) becomes

$$
\mathrm{d} F=-\Delta P \mathrm{~d} V_{\mathrm{b}}+\gamma(R) \mathrm{d} A
$$

This results in Manning's eqn (4)

$$
\mathrm{d} F / \mathrm{dR}=-4 \pi R^{2} \Delta P+8 \pi R \gamma(R)
$$

and, upon second derivation, in a stability criterion (Manning's eqn (8)) 


$$
\gamma(R)-R(\mathrm{~d} \gamma / \mathrm{d} R)<0
$$

As discussed above, the pressure exerted by the liquid to the bubble and the pressure exerted by the bubble to the liquid represent a pair of an action force and a reaction force, and they are there for the same reason. That reason is the surface free energy of the spherical gas-liquid interface. This free energy must be taken into account in the thermodynamic equations such as eqn (12). Physically, the situation related to an action force and a reaction force is symmetric, so that the surface free energy change of a bubble can be described either by the work done in changing the surface area, or the work done in changing the gas phase. Simple derivation shows that these terms are $\gamma(R) \mathrm{d} A=8 \pi R \gamma(R)$ and $-\mathrm{P}_{\mathrm{b}} \mathrm{d} V_{\mathrm{b}}=-8 \pi R \gamma(R)$. Thus, they are equal and opposite as they should, being works done by a pair of an action and a reaction force. The critical point here is that, in the thermodynamic equations of this system, the surface free energy change of a bubble must be taken into account only once.

Manning's ${ }^{1}$ eqn (2), i.e. our eqn (12) above, includes both the term $\gamma(R) \mathrm{d} A$ and the term $-P_{\mathrm{b}} \mathrm{d} V_{\mathrm{b}}$, as if they were separate free energies. This represents double-counting of the surface free energy of the bubble. A way to demonstrate this error is to insert eqn (11) into eqn (14), giving $\mathrm{d} F / \mathrm{d} R=0$. This suggests that the free energy of a volume of liquid including a bubble is independent of the size of the bubble. Clearly, this cannot be correct.

When the erroneous second term on the right hand side of eqn (12) is removed, the variable $-\Delta P$ will change to the variable $P_{\mathrm{w}}$, and the stability criterion in eqn (15) changes to

$$
P_{\mathrm{w}} R+\gamma(R)+R(\mathrm{~d} \gamma / \mathrm{d} R)>0
$$

Noting that $\mathrm{d} \gamma / \mathrm{d} R$ for a bubble should be positive, ${ }^{9,10}$ eqn (16) indicates that the bubbles, as considered by Manning, ${ }^{1}$ are always stable at a positive external pressure. This contradicts Manning's ${ }^{1}$ result and renders the analysis, based on the detailed dependence of $\gamma$ on $R$, meaningless.

Notwithstanding the criticism above, we agree with Manning ${ }^{1}$ in his assertion that the "Laplace Pressure Bubble Catastrophe" has no physical basis. A high pressure inside a bubble cannot logically be the cause of dissolving it, because in that case the pressure should decrease when the gas in the bubble dissolves and the bubble gets smaller while, according to the Young-Laplace equation, the pressure then increases. The bubble stability problem must be solved by considering the thermodynamics of a complete liquid, gas, vapour system while properly treating the free energy of the interface, and considering the energy barriers that may lead to metastable nanobubbles. ${ }^{11}$

\section{Conflicts of interest}

There are no conflicts to declare.

\section{Acknowledgements}

This work was funded by the Academy of Finland, grant no. 297278.

\section{References}

1 G. S. Manning, On the Thermodynamic Stability of Bubbles, Immiscible Droplets, and Cavities, Phys. Chem. Chem. Phys., 2020, 22(31), 17523-17531, DOI: 10.1039/D0CP02517H.

2 P. S. Epstein and M. S. Plesset, On the Stability of Gas Bubbles in Liquid-Gas Solutions, J. Chem. Phys., 1950, 18(11), 1505-1509, DOI: 10.1063/1.1747520.

3 S. Ljunggren and J. C. Eriksson, The Lifetime of a ColloidSized Gas Bubble in Water and the Cause of the Hydrophobic Attraction, Colloids Surf., A, 1997, 129-130, 151-155, DOI: $10.1016 /$ S0927-7757(97)00033-2.

4 C. A. Ward, P. Tikuisis and R. D. Venter, Stability of Bubbles in a Closed Volume of Liquid-gas Solution, J. Appl. Phys., 1982, 53(9), 6076-6084, DOI: 10.1063/1.331559.

5 L. Makkonen, On the Methods To Determine Surface Energies, Langmuir, 2000, 16(20), 7669-7672, DOI: 10.1021/ la990815p.

6 L. Dufour and R. Defay, Thermodynamics of Clouds, Acad. Press., New York-London, 1963, p. 69.

7 J. R. Taylor, et al., Classical Mechanics, University Science Books, 2005.

8 D. E. Brown, Students' Concept of Force: The Importance of Understanding Newton's Third Law, Phys. Educ., 1989, 24(6), 353.

9 Y. A. Lei, T. Bykov, S. Yoo and X. C. Zeng, The Tolman Length: Is It Positive or Negative?, J. Am. Chem. Soc., 2005, 127(44), 15346-15347.

10 S. Burian, M. Isaiev, K. Termentzidis, V. Sysoev and L. Bulavin, Size dependence of the surface tension of a free surface of an isotropic fluid, Phys. Rev. E, 2017, 95, 062801, DOI: 10.1103/PhysRevE.95.062801.

11 T. Vehmas and L. Makkonen, Metastable Nanobubbles, ACS Omega, 2021, 6, 8021-8027, DOI: 10.1021/acsomega. 0c05384. 